\title{
'Necessity is the mother of invention': Specialist palliative care service innovation and practice change in response to COVID-19. Results from a multinational survey (CovPall)
}

Palliative Medicine

2021, Vol. 35(5) 814-829

(C) The Author(s) 2021

(c) (1)

Article reuse guidelines:

sagepub.com/journals-permissions

DOI: 10.1177/0269216321100066

journals.sagepub.com/home/pmj

(S)AGE

\author{
Lesley Dunleavy ${ }^{1}$ (D) Nancy Preston ${ }^{1}$ (D), Sabrina Bajwah ${ }^{2}$ iD, \\ Andy Bradshaw ${ }^{3}$ (D) Rachel Cripps² ${ }^{2}$, Lorna K Fraser ${ }^{4}$ iD, \\ Matthew Maddocks ${ }^{2}$ iD, Mevhibe Hocaoglu², Fliss EM Murtagh ${ }^{3}$ iD, \\ Adejoke O Oluyase ${ }^{2}$ iD, Katherine E Sleeman² iD, Irene J Higginson² id \\ and Catherine Walshe ${ }^{1}$ iD; On behalf of the CovPall study team
}

\begin{abstract}
Background: Specialist palliative care services have a key role in a whole system response to COVID-19, a disease caused by the SARSCoV-2 virus. There is a need to understand service response to share good practice and prepare for future care.

Aim: To map and understand specialist palliative care services innovations and practice changes in response to COVID-19.

Design: Online survey of specialist palliative care providers (CovPall), disseminated via key stakeholders. Data collected on service characteristics, innovations and changes in response to COVID-19. Statistical analysis included frequencies, proportions and means, and free-text comments were analysed using a qualitative framework approach.

Setting/participants: Inpatient palliative care units, home nursing services, hospital and home palliative care teams from any country. Results: Four hundred and fifty-eight respondents: 277 UK, 85 Europe (except UK), 95 World (except UK and Europe), 1 missing country. $54.8 \%$ provided care across $2+$ settings; $47.4 \%$ hospital palliative care teams, $57 \%$ in-patient palliative care units and $57 \%$ home palliative care teams. The crisis context meant services implemented rapid changes. Changes involved streamlining, extending and increasing outreach of services, using technology to facilitate communication, and implementing staff wellbeing innovations. Barriers included; fear and anxiety, duplication of effort, information overload and funding. Enablers included; collaborative teamwork, staff flexibility, a pre-existing IT infrastructure and strong leadership.

Conclusions: Specialist palliative care services have been flexible, highly adaptive and have adopted low-cost solutions, also called 'frugal innovations', in response to COVID-19. In addition to financial support, greater collaboration is essential to minimise duplication of effort and optimise resource use.
\end{abstract}

ISRCTN16561225 https://doi.org/10.1186/ISRCTN16561225

\section{Keywords}

Palliative care, hospice, coronavirus infections, surveys and questionnaires, pandemics, COVID-19

What is already known about the topic?

- Specialist palliative care services are part of a whole healthcare system response to COVID-19 which involves services working collaboratively with each other and with other external health care organisations in response to the pandemic.

- Services need to make practice changes in response to the global pandemic.

\footnotetext{
${ }^{1}$ International Observatory on End of Life Care, Lancaster University, Lancaster, UK

${ }^{2}$ Cicely Saunders Institute of Palliative Care, Policy and Rehabilitation, King's College London, London, UK

${ }^{3}$ Wolfson Palliative Care Research Centre, Hull York Medical School, University of Hull, Hull, UK

${ }^{4}$ Martin House Research Centre, Department of Health Sciences, University of York, UK
}

Corresponding author:

Lesley Dunleavy, International Observatory on End of Life Care, Faculty of Health and Medicine, Division of Health Research, Health Innovation One, Sir John Fisher Drive, Lancaster University, Lancaster, LA1 4AT, UK. Email: I.dunleavy@lancaster.ac.uk 


\section{What this paper adds}

- Specialist palliative care services responded rapidly to COVID-19 in both planning for change and then adapting to needs and requirements.

- Services often relied on 'improvisation', 'quick fixes' and 'making do' when responding to the COVID-19 crisis.

Implications for practice, theory or policy

- In addition to financial support, greater collaboration is essential to build organisational resilience and drive forward innovation, by minimising duplication of effort and optimising resource use.

- The effectiveness and sustainability of any changes made during the crisis needs further evaluation.

\section{Background}

The COVID-19 pandemic, and the healthcare service response to this, is an example of a so-called 'wicked problem', constantly changing, difficult to define and with multiple interdependencies ${ }^{1}$; what the army would call a VUCA situation: 'volatile, uncertain, complex and ambiguous'. ${ }^{2}$ Adaptations, flexibilities and innovative practices are necessary ${ }^{3}$ in this crisis context. This includes innovative responses of healthcare systems, where hospice and palliative care services are an integral part of such a response to the COVID-19 pandemic.

The term innovation can have multiple meanings and be discipline specific. ${ }^{4}$ Broadly, innovations are the tools used by organisations to influence or respond to environmental change and can encompass both radical and incremental innovation. ${ }^{4,5}$ In healthcare, innovation has been defined as; 'a novel set of behaviours, routines, and ways of working that are discontinuous with previous practice. . . and that are implemented by planned and coordinated actions' (p.582). ${ }^{6}$ A more ubiquitous definition, also used in healthcare, ${ }^{7,8}$ defines innovation more broadly as 'an idea, practice or object that is perceived as new' so a change in practice may be novel even if the same approach has been used elsewhere (p.12). ${ }^{9}$ In crisis management, innovation can incorporate 'improvisation', 10-13 which involves organisations using, adjusting and recombining existing resources, structures and processes to manage the impact of a crisis. ${ }^{14}$ In this paper, the term innovation is used as a broad umbrella term that includes 'improvisation' and practice change.

Practice changes that may be regarded as innovations against these definitions are likely to be required and seen in response to COVID-19, especially with rapidly shifting priorities, new learning about the disease, potential shortages of drugs and equipment, and adjusting to workforce pressures and redeployments. ${ }^{15} \mathrm{~A}$ large number of deaths have been associated with COVID-19 so there is need to understand the role specialist palliative care services play in providing end of life care in this context. ${ }^{16}$ Commentaries indicate an initial rapidity of service changes with reports of new staffing and service delivery models, virtual care and addressing shortages all in the context of infection control procedures and heightened fear and anxiety. ${ }^{17-21}$ It is imperative that we consider which might be sustained as part of a 'new normal', and which may quietly fall away, ${ }^{22,23}$ with a focus on a learning mindset. ${ }^{24}$ In this context, it is important that there is wide learning about how hospice and palliative care services have responded to the COVID-19 pandemic, so that effective innovations can be rapidly shared, and preparations made for future care, including second or third waves or other pandemic or emergency situations. ${ }^{25}$

\section{Methods}

\section{Aim}

To map and understand specialist palliative care services innovations and practice changes in response to the COVID-19 pandemic. This paper is part of the wider CovPall study that aims to understand the breadth of the multinational specialist palliative care response to COVID-19, ${ }^{26}$ including clinical palliative care of those with COVID-19.

\section{Design}

An online multinational cross-sectional survey of hospice and specialist palliative care providers. This study is reported according to the STROBE ${ }^{27}$ and CHERRIES $^{28}$ statements.

\section{Population and setting}

Inpatient palliative care units, hospital palliative care teams, home palliative care teams and home nursing services were eligible to provide data, from any country (see Supplemental materials for definitions).

\section{Sampling and recruitment}

The aim of the recruitment strategy was to receive responses from all hospice and specialist palliative care services. Information was widely disseminated through key collaborators (e.g. Hospice UK, Marie Curie Care, Sue Ryder Foundation, European Association of Palliative 
Table 1. Free text response survey questions reported in this paper.

Question 4.2-4.3: Would you say overall you are more busy or less busy than before the COVID-19 pandemic? and why is this?

Question 4.11: What have been the difficulties of using virtual technologies?

Question 4.12: What has worked well when using virtual technologies?

Question 4.18: What would you say are the main challenges for bereavement support during the COVID-19 pandemic?

Question 4.19: How are you supporting patients with COVID-19 who are from more disadvantaged socio-demographic communities (e.g. areas with greater poverty, poor housing, homelessness)?

Question 4.21: Are there any groups (e.g. with different religions, cultures) where you have found supporting the individual needs of people affected by COVID-19 is particularly challenging?

Question 4.22: How has COVID-19 changed how you are supporting the types of patients (e.g. with symptoms and progressive illness) that you would usually support?

Question 4.23: How has COVID-19 changed how you are supporting the families/those important to patients that you would usually support?

Question 5.1a: What changes were there in how you used your beds (if any)?

Question 5.1c: Any changes to admission criteria (if so what was the change)?

Question 5.1d: Any changes to out of hours admissions (e.g. evenings/weekends - if so what was the change)?

The three questions below were asked for acute hospitals, home support and care homes

Question 5.1i/p/x: Have you changed how your team is organised (e.g. supporting patients with and without COVID-19)?

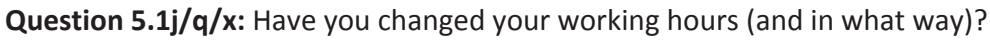

Question 5.1k/r/\&: Have you changed your working practices (and in what way)?

Question 5.1s-5.1t: Have you changed how medicines are given in the community (e.g. who sets up syringe drivers/families administering medicines)? (if yes - a box for details will open) Please give details (changed how medicines are given in the community)

Question 5.3-5.3 a: Have you changed how you contact and work with families/those important to patients? (If yes, a box for details will open) Please give details (changed how contact and work with families)

Question 5.5-5.5 a: Have you changed how you deploy volunteers? (if yes - a box for details will open) Please give details

Question 6.9-6.10: Please tell us about the change in practice or innovation that you think has been most successful to your working. Why is this?

Question 6.11: What would you say were the most important things that made this possible?

Question 6.12: Please list any other important changes/innovations you have made

Care), contacts through publicly available service directories, information provided on institutional websites, personal networks, and via the social media channels of investigators and key collaborators. Potential participants could contact the study team to receive a participant information sheet and link to complete the survey online. The clinical lead or their nominee completed the survey.

\section{Data collection}

REDCap was used to securely build and host the online survey with closed and free text survey responses (see Supplemental materials for the full survey). Sites could enter the data online directly (with a pause and return function), be sent the survey as a word document via email to complete and return electronically, or request to answer the survey questions via telephone or video conferencing with a member of the study team. Multiple questions with free-text response options within the survey addressed relevant areas for this analysis, and are presented in Table 1.

\section{Data analysis}

Anonymised data were exported to SPSS (for quantitative analysis using descriptive statistics, frequencies, proportions and means) and NVivo 12 (for analysis of free-text comments using a qualitative framework analysis approach). ${ }^{29}$ Continuous variables were expressed as means (SD) and medians (IQR) and categorical variables as counts and percentages. Missing data were not imputed. An analytical framework was initially developed by LD and CW through familiarisation with the data, the framework was then applied to the free text data and refined, as appropriate, during the analysis process. LD and CW used analytical memos and charting to aid interpretation of the data.

\section{Research ethics and approvals}

Research ethics committee approval for this study was obtained from King's College London Research Ethics Committee (21/04/2020, Reference; LRS19/20-18541). The study was registered on the ISRCTN registry (27/07/2020, ISRCTN16561225).

\section{Findings}

The survey was open to responses from 23/04/2020 to $31 / 07 / 2020$. Responses were received from 458 respondents: 277 UK, 85 Europe (except UK), 95 World (except UK and Europe), 1 missing country. The response rate could 
not be calculated as the survey denominator was unknown. Table 2 reports data on the characteristics of responding services and answers to the survey questions explored in the findings below (see Supplemental materials for details of services offered before the pandemic discussed in the findings below).

The overarching categories identified in the analysis included 'the crisis context', the changes made (streamlining access, extending services, increasing outreach, using communication technology and implementing innovations for staff wellbeing) and the enablers and barriers for change (see Figure 1). These were identified from services responding from across the world, and often where they provided care across a range of different service types. As discussed previously, the term innovation is used as a broad umbrella term that includes 'improvisation' and practice change. Exemplar data extracts for each category and subcategory are presented in Table 3 and in the narrative below, along with supporting quantitative data.

\section{The crisis context}

All services had to change, often rapidly, to prepare for, and respond to, the anticipated and actual impact of the COVID-19 pandemic. Services often initiated changes that had been previously considered but rejected or resisted.

\section{Streamlining access to specialist palliative care services}

\section{Specialist palliative care triage and assessment/single point of access}

The pandemic led to changes in how referrals to services were received, assessed and managed, both initially and on an ongoing basis. This included, for example, proactively seeking referrals, loosening or tightening referral criteria, such as not accepting patients for respite care, and the use of telephone advice lines:

\footnotetext{
'Setting up a regional single point of access with two other local hospices and pooling resources so patients, families and HCPs [healthcare professionals] have one number to call to gain advice, support or rapid discharge, admission to a hospice etc.' 'Something we have been working towards for a long time, but very slowly. The pandemic has allowed us to set it up in 10 days and iron out problems as we've gone. People have been a lot more collaborative.' (participant 92, UK, adult, inpatient palliative care unit/home palliative care team)
}

How referrals for people without COVID-19 and with confirmed or suspected COVID-19 were dealt with was based on both clinical need and how to manage the risk of infection for patients, family carers and staff:
'Triage telephone assessment prior to visits and in maintaining on going symptom management support.' 'It has enabled scrutiny of issues to prioritise a visit.' (participant 47, UK, paediatric, inpatient palliative care unit/home palliative care team)

Telephone calls were used to keep in regular contact with patients and family carers including proactive calls for patients who were stable but isolated:

'Calls to all patients to gauge vulnerability, how they would get meds/ shopping in lockdown, what their supports were and specific form for this completed to help us know who to focus on- these patients received extra connection calls and support.' (participant 421, rest of world (HIC), adult, inpatient palliative care unit/home palliative care team/ home nursing services)

Ongoing telephone support would also be provided for those patients who were unable to attend outpatients or day therapy services because of restrictions. Services wanted to reassure patients and family carers that 'the care is still here, it just looks different' (participant 42, UK, adult, home palliative care team).

\section{Extending current specialist palliative care services}

\section{Inpatient bed management}

$41.3 \%$ of services who had changed in response to COVID19 reported changes in inpatient beds in their service with $27.6 \%$ reporting an increase in bed numbers. This was often in situations where dedicated beds previously did not exist, or else increasing capacity by using space in flexible ways:

'Having palliative care beds in the hospital, supported by palliative care doctors' 'early lobbying for a palliative care ward from palliative care team.' (participant 61, UK, adult, hospital palliative care team)

'We re-configured our well-being centre to provide 12 extra IPU [In-patient unit] beds. We have stopped providing respite during the pandemic to open up beds. We are admitting routinely 7 days per week.' (participant 94, UK, adult, inpatient palliative care unit/home palliative care team/ home nursing services)

When reconfiguring inpatient areas, services needed to take account of 'hot' areas or zones where those with suspected or confirmed COVID-19 were cared for and 'cold areas' where people not suspected of having COVID-19 were cared for:

In patient area in acute hospital repurposed as COVID cohort ward for end of life care; second ward in community hospital 


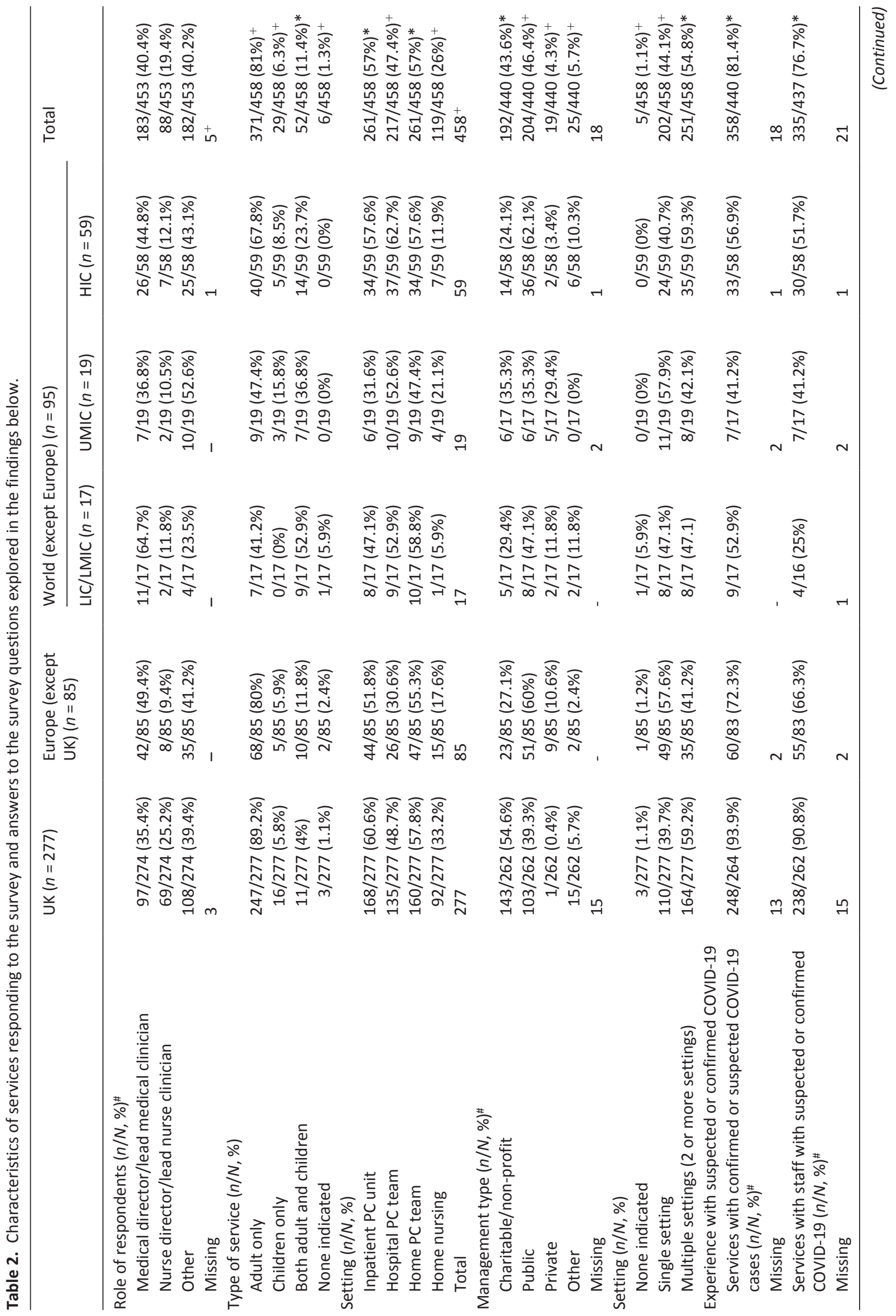




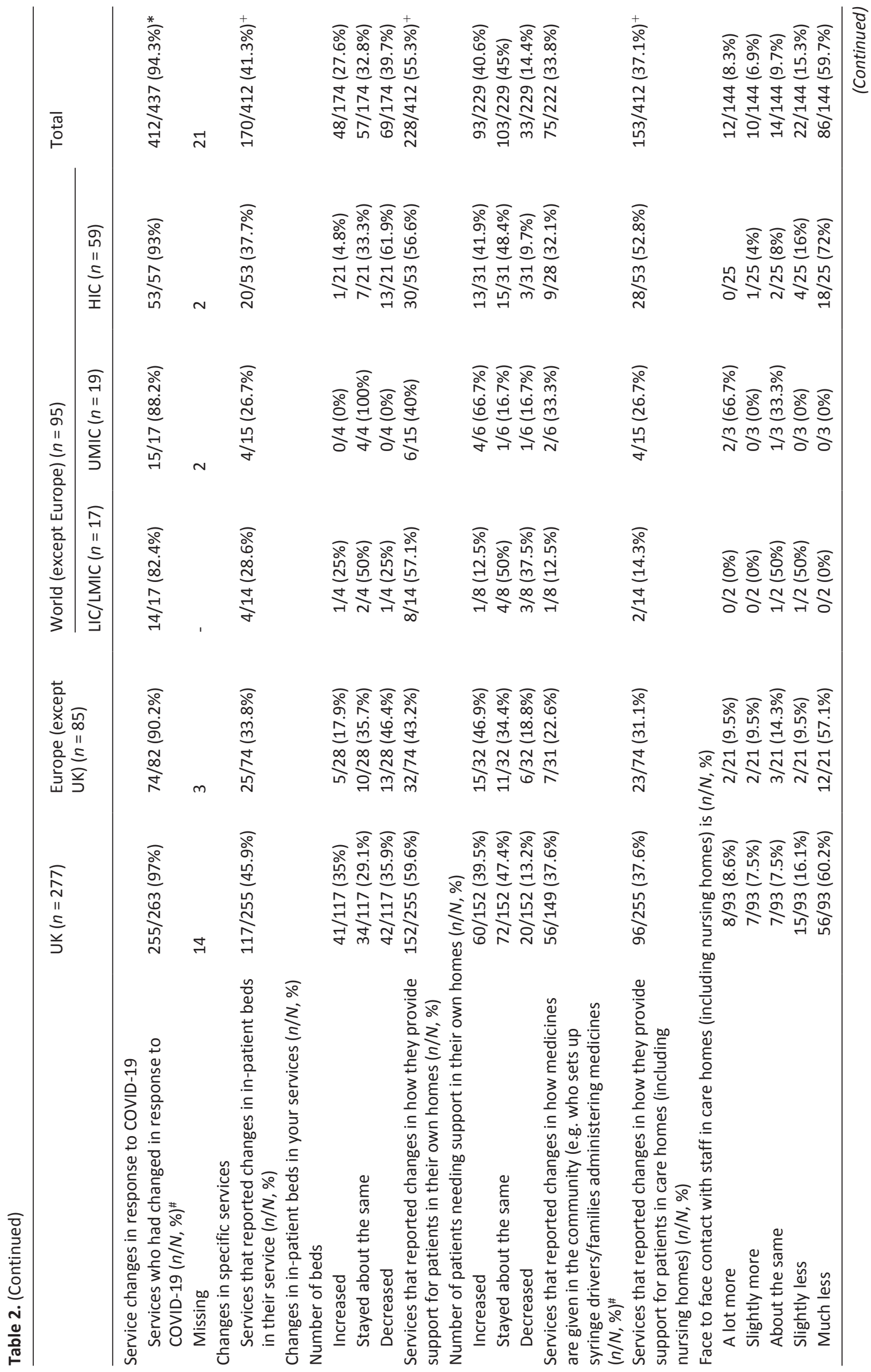




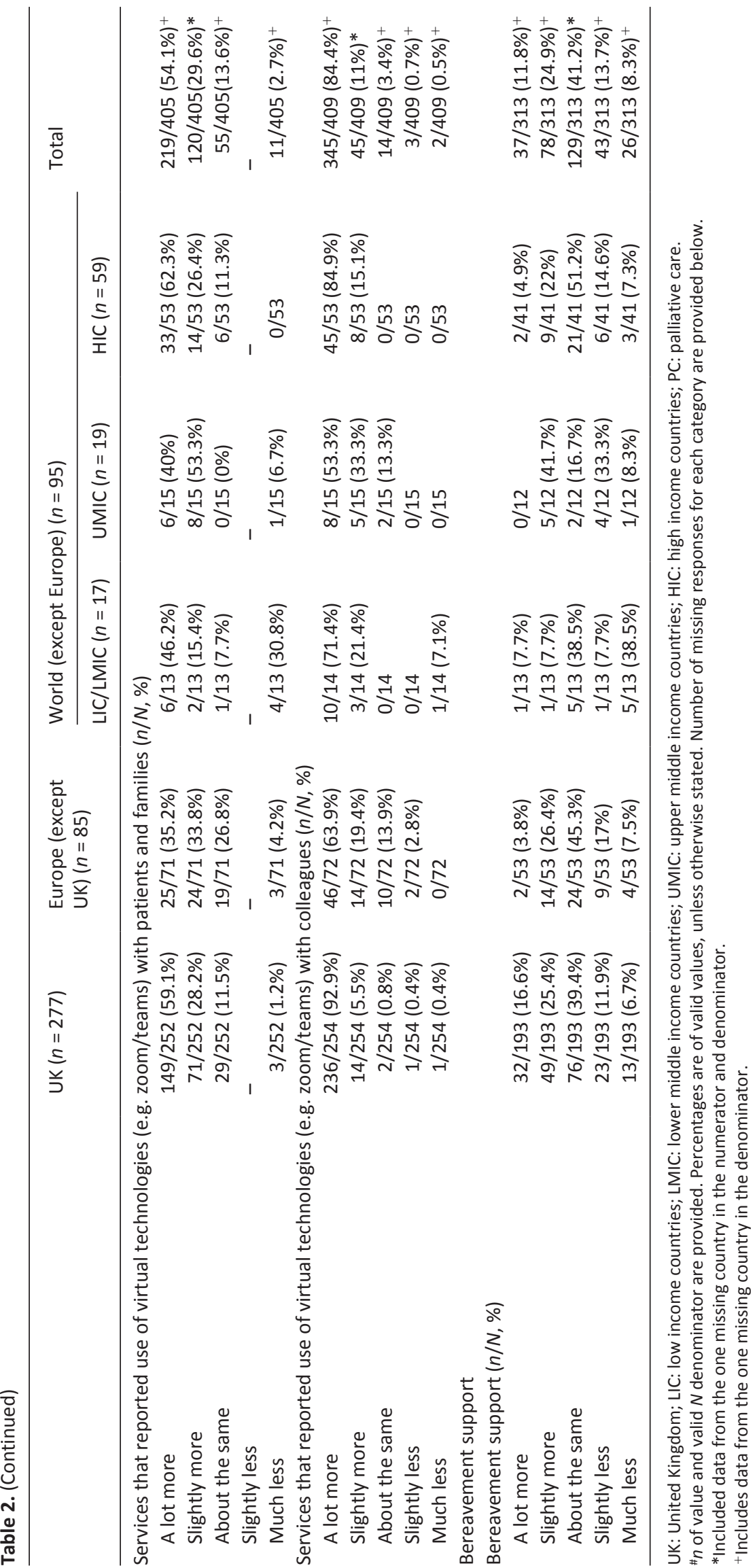




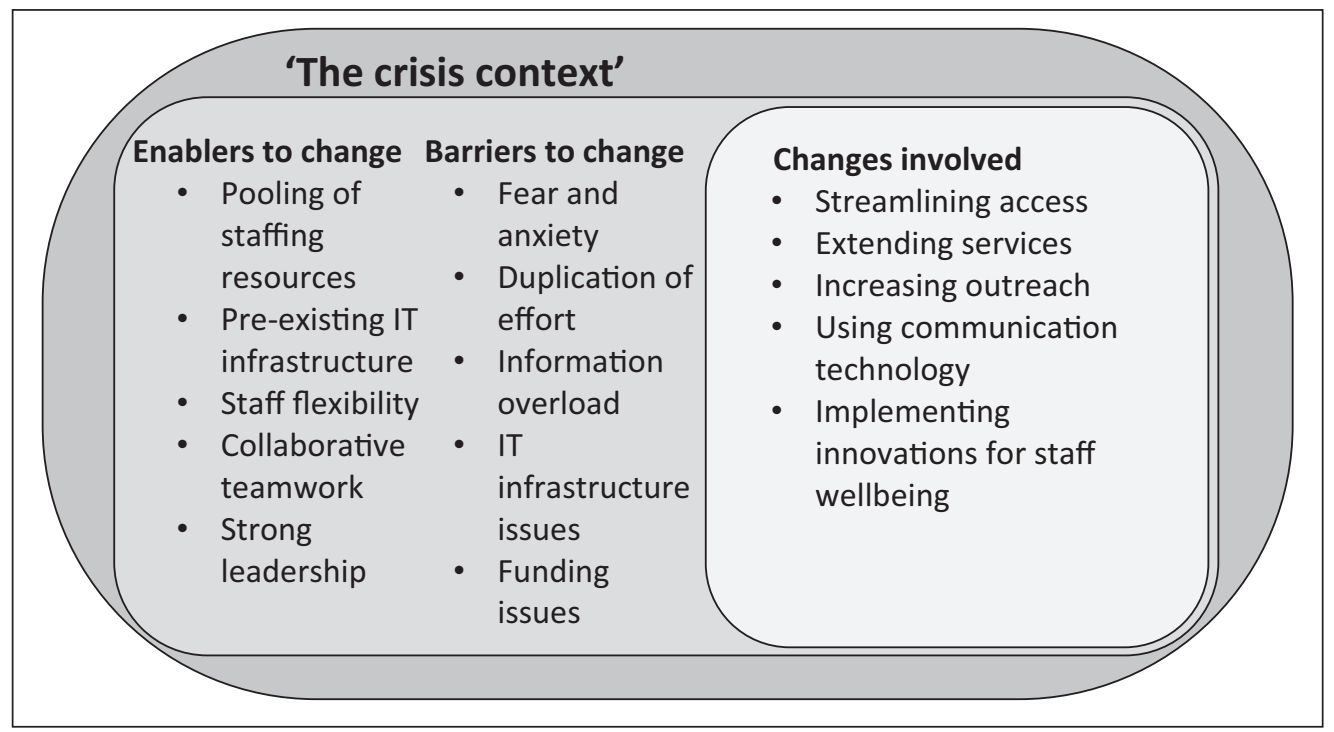

Figure 1. Overarching analytical categories.

Table 3. Categories and sub-categories.

\begin{tabular}{lll}
\hline Category & Sub category & Illustrative data extracts \\
\hline 'The crisis context' & $\begin{array}{l}\text { Necessity-risk } \\
\text { management }\end{array}$ & $\begin{array}{l}\text { 'The crisis forced change that had been resisted pre-COVID. For the education } \\
\text { service, the crisis meant that they either changed to virtual working or the ser }\end{array}$
\end{tabular}
would close. This has opened up the reach of the team. Redeployment was resisted but COVID meant it became a necessity.' (participant 339, UK, adult, inpatient palliative care unit/home palliative care team/home nursing services)

Streamlining access to specialist palliative care services

Specialist palliative care triage and assessment

Single point of access

Extending current specialist palliative care services
Inpatient bed

management

Support in the community

Specialist palliative care support out of hours

Bereavement services

Management of medicines and medication administration
'Potential admissions to hospital all go via Blue Line - staffed by experienced clinicians but put through to me if palliative. . Involved in decision making prior to admission means patients come in with clear plan and potential shorter admission time.' (participant 135, UK, adult, hospital palliative care team/home palliative care team)

'Created a clinical Coordination service which manages all incoming referrals and calls for all of our services, with staff rotating into these services.' (participant 36, $U K$, adult, inpatient palliative care unit/home palliative care team/home nursing services)

'Support is there and our response is in place (as yet untested and not needed to the fullest extent in IPU bed provision).' (participant 157, UK, adult, inpatient palliative care unit/hospital palliative care team/home palliative care team) 'We combined both community teams (usually split according to geography) as we were largely working remotely and to enable larger numbers of patients to be contacted.' (participant 110, UK, adult, inpatient palliative care unit/home palliative care team/home nursing services)

'Changes to usual practice to ensure increased availability of medical palliative care cover 27/7; 7 day CNS working already in place.' (participant 33, UK, adult, hospital palliative care team)

'Now all families in our area are eligible, regardless of whether they are known to the hospice. Support is done remotely by telephone/video call, which can be less satisfying compared to face to face.' (participant 386, UK, adult, inpatient palliative care unit, home nursing services).

'We created a symptom management 'order set' in our electronic prescribing system. This was based on the APM guidance for Covid-19. It gave guidance for prescribers of what drugs to choose for what symptoms and encouraged them to co-prescribe anti-emetics and laxatives where needed.' (participant 208, UK, adult, hospital palliative care team/home palliative care team) 
Table 3. (Continued)

\begin{tabular}{|c|c|c|}
\hline Category & Sub category & Illustrative data extracts \\
\hline \multirow[t]{2}{*}{$\begin{array}{l}\text { Increasing } \\
\text { outreach to } \\
\text { generalist services }\end{array}$} & $\begin{array}{l}\text { Specialist palliative } \\
\text { care outreach into the } \\
\text { hospital }\end{array}$ & $\begin{array}{l}\text { 'we had to move our unit to another building, we were tasked to write protocols } \\
\text { for the COVID units in case of palliative sedation, pain management, anxiety } \\
\text { management. . . and protocols with alternative drugs in case midazolam was } \\
\text { unobtainable and our advisory team worked in the COVID units regularly on } \\
\text { request.' (participant 392, Europe, adult, inpatient palliative care unit/hospital } \\
\text { palliative care team) }\end{array}$ \\
\hline & $\begin{array}{l}\text { Specialist palliative } \\
\text { care outreach into the } \\
\text { community }\end{array}$ & $\begin{array}{l}\text { 'using ZOOM to do multidisciplinary rounds/mortality rounds with nursing homes } \\
\text { and also to replace visits for cases who are not actively dying or having symptoms } \\
\text { that are not too complex. - tele-training of } \mathrm{NH} \text { [nursing homes] nurses' (participant } \\
\mathbf{2 3 9} \text {, rest of world (HIC), adult, home palliative care team/home nursing services) }\end{array}$ \\
\hline \multirow[t]{3}{*}{$\begin{array}{l}\text { Using } \\
\text { communication } \\
\text { technology }\end{array}$} & $\begin{array}{l}\text { Using communication } \\
\text { technology with patients } \\
\text { and family carers }\end{array}$ & $\begin{array}{l}\text { 'More use of Near-Me tele-medicine.' 'Near me saves patients coming to inpatient } \\
\text { clinics. This could be used more after the Covid situation has passed, as often our } \\
\text { patients struggle to come to clinic.' (participant 46, UK, adult, inpatient palliative } \\
\text { care unit/hospital palliative care team/home palliative care team) }\end{array}$ \\
\hline & Virtual visiting & $\begin{array}{l}\text { 'On the COVID unit we only allowed } 2 \text { visitors for } 30 \text { min to say goodbye to a dying } \\
\text { patient. We invested a lot in virtual saying goodbye with Jitsi (IT installed special } \\
\text { laptops for this). We have a very good team of psychologists and chaplains that } \\
\text { were available } 24 / 7 \text { to care for family, to give support to the COVID team and } \\
\text { helped with the virtual goodbye saying.' (participant 186, Europe, adult, inpatient } \\
\text { palliative care unit/hospital palliative care team) }\end{array}$ \\
\hline & $\begin{array}{l}\text { Using technology to } \\
\text { facilitate communication } \\
\text { between healthcare }\end{array}$ & $\begin{array}{l}\text { 'Daily updates to all staff - if staff unable to attend in person this is relayed via } \\
\text { WhatsApp group messaging so all staff are informed of the same information.' } \\
\text { (participant 51, UK, adult, inpatient palliative care unit/home nursing services) }\end{array}$ \\
\hline
\end{tabular}

Implementing

innovations for

staff well-being

Enablers
Pre-existing IT

infrastructure

Pooling of staffing resources

Staff flexibility (both a willingness and a need to be flexible)

Strong leadership

Collaborative teamwork (within and between specialist palliative care services and with other generalist palliative care providers).

Fear and anxiety (patient, family carer and healthcare professional)

Duplication of effort
2. Resilience - we helped Covid-19 teams from intensive care and emergency room with that aspect by providing them with complementary medical treatments such as acupuncture and other touch therapies such as Reflexology and Shiatsu. These were provided by our integrative oncology team members. (participant 478, rest of world (HIC), adult, hospital palliative care team)

'We were very fortunate to have a well-supported electronic patient record system with the necessary IT hardware to support it. This was fundamental in allowing us to work remotely.' (participant 206, Europe, adult, home palliative care team) 'We have redeployed a number of staff to different services and paired them up with more experienced staff members who have shared their skills and supported their development.' (participant 5, UK, adult, inpatient palliative care unit/ hospital palliative care team/home palliative care team/home nursing services) 'In difficult times, most people give the best and there is no time to watch those who are always complaining' (participant 316, Europe, adult, inpatient palliative care unit)

'Leadership working above and beyond, to resolve PPE and supply shortages, and finding solutions to keep staff, patients and families safe.' (participant 383, rest of world (HIC), adult, home palliative care team/home nursing services)

'Ongoing collaboration between SPC [specialist palliative care] in local area which has been essential as we learn from each other and assist one another in managing eg sharing plans, guidance, telephone numbers and contacts. Excellent pre-existing relationships with other local providers has meant we can draw on those links to assist in this crisis.' (participant 192, UK, adult, inpatient palliative care unit, home nursing services).

'We have to be more careful and adopt more severe protective measures for our patients and ourselves. Besides, we need to handle the emotional consequences that the pandemic has caused (tension, fear, suspicion . . .) on our staff.' (participant 486, Europe, adult, inpatient palliative care unit/hospital palliative care team/home palliative care team/home nursing services)

'Number of cases has not especially gone up and reduced in some areas. But busy due to planning, keeping up to date with change in polices, creating new guidance and pathways.' (participant 151, UK, adult/paediatrics, inpatient palliative care unit/ hospital palliative care team/home palliative care team/home nursing services) 
Table 3. (Continued)

\begin{tabular}{lll}
\hline Category & Sub category & Illustrative data extracts \\
\hline Information overload & $\begin{array}{l}\text { 'Busy adjusting to constantly changing guidelines/anxieties from staff etc' } \\
\text { (participant 130, rest of world (HIC), adult, hospital palliative care team/home } \\
\text { palliative care team) }\end{array}$ \\
'Zoom is a security risk. data is super expensive. not all staff have fiber network \\
set up at home. staff are all not fully IT literate, fear of using technology, poor \\
network.' (participant 28, rest of world (UMIC), adult, inpatient palliative care \\
unit/home palliative care team)
\end{tabular}

previously used as 'hospice' reassigned as 'clean' palliative care area.' (participant 7, UK, adult, inpatient palliative care unit/ hospital palliative care team/home palliative care team)

Some also reported that inpatient bed numbers stayed the same, decreased and that additional beds were not always needed.

\section{Support in the community}

$55.3 \%$ of services that had changed due to COVID-19 said there had been changes in how they provided support for patients in their own home with $40.6 \%$ saying the number of patients needing support at home had increased. There was a shift in patient need from the inpatient to the community setting in some areas:

'We have amalgamated a number of different teams i.e. CNS [Clinical Nurse Specialist], H@H [Hospice at Home], AHP [Allied Health Professionals], IPU [Inpatient Unit] into 3 community locality multi-professional teams. These provide both specialist care and in addition, personal care for anyone in the last 3 months of life. This means that any face to face visiting might occur for instance between a CNS [Clinical Nurse Specialist] and a HCA [Health Care Assistant] so that both forms of specialist and personal care can be given. This reduces footfall within patient's homes. It also helps with staffing.' (participant 235, UK, adult, inpatient palliative care unit/home palliative care team/home nursing services)

\section{Specialist palliative care support out of hours}

Some respondents described extending their current out of hours medical and/or nursing provision during the peak of the pandemic to support generalist palliative care providers and facilitate hospice inpatient admissions out of hours:

'We moved to a 7 day onsite service to be able to see patients that were referred at weekends and who had significant symptoms deteriorating quickly. This also allowed up to check in with areas of the hospital who were dealing with larger number of deaths than normal and try support patients and staff early were possible.' (participant 351, UK, adult, hospital palliative care team)

Medical and nursing shift patterns were sometimes adjusted to accommodate the increase in out of hours provision. Some services reported that routine hours of working had resumed in their locality.

\section{Bereavement services}

$36.7 \%$ of respondents reported that they were providing slightly more or a lot more bereavement support than before the pandemic with $41.2 \%$ offering about the same. Some were offering support to those not directly under the care of the specialist palliative care service, including to nursing home staff, and educating others on how to provide bereavement care:

'reorganising the family support team to work virtually and provide more education and support to wider groups outside of the hospice. e.g support for staff outside of the hospice on how to support someone with bereavement.' (participant 194, UK, adult, inpatient palliative care unit/home palliative care team/home nursing services)

Services were starting to see referral numbers pick up and were planning for the anticipated increase in those requiring bereavement support as a result of the pandemic.

\section{Management of medicines and medication administration}

The pandemic led to changes in how medicines were managed with routine practices and processes adapted to reduce infection risk and unnecessary visits, such as single use syringe drivers and specialist palliative care professionals administering medication in services where this was not norm. New processes were set up to improve access to symptom control medication: 
'Palliative care team now administering medications in the home where previously this was only done by district nurses. Grab Bags available for prescribers to take out medications to the house for administration (not to be left in the house). Now have PGDs (Patient Group Directives) that enable nonprescribers to take anticipatory medications out on visits in a Grab Bag and administer up to 3 PRN doses of Morphine/ Midazolam/Buscopan//Levomepromazine under specific circumstances.' (participant 367, UK, adult, inpatient palliative care unit/hospital palliative care team/home palliative care team)

$33.8 \%$ of services who had changed how they provided support for patients in their homes said they changed how medicines were given in the community. In those areas where policies were not already in place, policies were developed to support carer administration of subcutaneous medication and/or routes of administration were changed to oral.

\section{Increasing outreach to generalist palliative care services}

\section{Specialist palliative care 'outreach' into the hospital}

Hospital specialist palliative care teams often shifted from a responsive to a proactive model of care as patients with COVID-19 could deteriorate and die rapidly and some of those providing direct care lacked end of life care experience. Teams proactively engaged with clinicians in areas where COVID-19 patients were being cared such as intensive care units, emergency departments and respiratory wards. They focused on; developing and disseminating COVID-19 symptom control guidelines, providing symptom control advice, supporting colleagues with complex treatment escalation or withdrawal decisions, visiting patients as necessary, and providing end of life care training and support including how to communicate with relatives over the telephone. Training and guidelines needed to be brief and rapidly developed:

'Staff on wards caring for patients with coronavirus identified that the biggest challenge was communicating with relatives over the telephone. Our hospital specialist palliative care team, led by the registrar, developed one-page guides to support this' (participant 31, UK, Adult, inpatient palliative care unit/hospital palliative care team)

\section{Specialist palliative care 'outreach' into the community}

$59.7 \%$ of services who had changed how they provided support for patients in care homes (including nursing homes) reported that their face-to-face contact with care home staff was much less during this time. Prior to
COVID-19 only $27.7 \%$ of respondents provided telehealth/video support/e-learning for education. Specialist palliative care services proactively contacted care homes to offer support and advice and education was also provided from a distance:

'They [medical staff] have set up twice weekly webinars for the wider health community, including GPs, nurses and nursing homes as well as a new advice email for GPs.' (participant 330, UK, Adult, inpatient palliative care unit/ home palliative care team)

\section{Using communication technology}

Services were forced to adopt the use of technology so that some clinical services could continue to operate. Patients could decline inpatient admission or face-to-face visits as they were fearful of contracting the virus and were concerned about the visiting restrictions. Some clinical staff, including those who were shielding, worked from home when able, to maintain social distancing.

\section{Using communication technology with patients and family carers}

Prior to COVID-19, only $21.6 \%$ of services used telehealth/video support/e-learning for clinical care with $83.7 \%$ of services that changed due to COVID-19 reporting that they were using virtual technologies with patients and families a lot more or slightly more during the pandemic. Generic digital platforms were used for communication such as Zoom, Skype, WhatsApp and Facebook. Hospice day therapy services were also provided off site using this technology such as complementary therapies via Skype and 'Time to create' via a Hospice YouTube channel. There were also reports of telemedicine being used, electronic care plans and applications to facilitate symptom assessment, virtual ward rounds and admission assessments:

. . our physician has been making virtual rounds which have been very successful through the use of the app.' 'We were well set up to use technology for reporting patient care, as well as general updates. During the COVID pandemic, this allowed us to maximize the use of this app to extend it to allowing for virtual rounds with the doctor, as well as to complete virtual intake assessments with referrers.' (participant 218, rest of world (HIC), adult, inpatient palliative care unit)

Using digital technology could be challenging especially with those who were hard of hearing, very sick or not previously known to the team. Not everyone had access to a computer, smart phone or the internet and connectivity could be an issue in rural areas. Some patients, particularly older people, were not keen to engage with digital technology. Prior to COVID-19, 66.2\% of services already 
used telephone support for clinical care and respondents reported that its use increased during the pandemic:

'There has been more telephone contact with our usual community patients, either due to our choice or the patient's. This has been ok but not ideal especially for difficult conversations or for new patients. It has however been more time efficient.' (participant 19, UK, adult, hospital palliative care team/home palliative care team)

Lack of closeness and human contact were reported as issues with remote working. Volunteer befriending, bereavement support and hospice day therapy services were also provided by telephone.

\section{Virtual visiting}

The pandemic led to visiting restrictions being imposed within inpatient units which caused distress to patients, family carers and staff members. Services utilised technology to facilitate 'virtual visits' and ensure lines of communication between patients and carers were kept open:

'The iPads which we managed to raise through charity donations - through the use of our Face Book page - are now available on all wards with the support of our IT team and IG teams have allowed many families to speak or even just see their loved ones.' (participant 175, UK, adult, hospital palliative care team)

A less costly and simpler strategy was the use of postcards for e-mail and telephone messages from relatives to be given or read out to patients.

\section{Using technology to facilitate communication between healthcare professionals}

$84.4 \%$ of services that changed in response to COVID-19 reported that they were using virtual technologies (e.g. zoom/teams etc.) with colleagues a lot more than before the pandemic. It was used to facilitate communication within specialist palliative care teams, across specialist palliative care services and with generalist clinicians and external partner organisations. Respondents felt the benefits included increased efficiency by reducing travel time, keeping the team connected and up to date with the everchanging situation as well as helping to facilitate the support process:

'firstly the support we gave our teams on a daily level. we realized they were leaving their family at home to visit patients and we perceived that as a vulnerable situation to many team members. we compiled a extensive reaching out plan to all the teams on an individual, sectorial, regional and nationwide level with phone support and zooms by professional management and general management.' (participant 247, rest of world (HIC), adult/paediatric, inpatient palliative care unit/hospital palliative care team/home palliative care team)

Using technology required an understanding of virtual meeting 'etiquette' and could be exhausting with one respondent describing it as 'Zoom fatigue' (participant 222, rest of world (HIC), adult/paediatric, home palliative care team).

\section{Implementing innovations for staff well being}

$76.7 \%$ of services had staff with suspected or confirmed COVID-19 so services needed to manage the impact of COVID-19 on staff wellbeing:

'It has been awful to witness the sorrow and pain of some families, when they have been separated in times of crisis.' (participant 225, Europe, adult, hospital palliative care team)

Different strategies were implemented to promote staff well-being while ensuring social distancing. The importance of checking in with staff on a regular basis, including those furloughed, to keep them informed of the everchanging situation, and provide opportunities for staff debriefing was reported. More practical strategies included; free car parking and meals, help with child care and virtual yoga or complementary therapy:

'Our hospice clinic room has been repurposed as a staff room - 'the bubble' - again this is something we'd been talking about for several months but had been hard to agree on where it would be; in the event two colleagues did a makeover of the room one day, including soft furnishings and hand creams / handmade scrubs bags, handmade fixtures for masks to avoid skin irritation, chocolate etc - feels important as one way to show colleagues are valued.' (participant 128, UK, adult, inpatient palliative care unit/hospital palliative care team/home palliative care team)

\section{Enablers and barriers to change}

Changes in practice occurred in a crisis context and in some instances this accelerated changes that had been previously planned or hoped for:

'Necessity is the mother of invention. The situation has forced us to be creative and some of the ways we have done this will stay with us post covid.' (participant 428, UK, adult, inpatient palliative care unit/home palliative care team/home nursing services)

'Streamlining of two services managed by different organisations (acute trust and hospice) which we have wanted 
to achieve for decades!' 'Appetite for change on both sides to work together and disregard former barriers to put the interests of patients first - long may it last!' (participant 388, UK, adult, inpatient palliative care unit/home palliative care team/home nursing services)

Respondents identified several factors that they felt enabled the imposed changes to be implemented swiftly into clinical practice. These included; pooling of staffing resources, staff flexibility (both a willingness and a need to be flexible), strong leadership, collaborative teamwork (within and between specialist palliative care services and with other generalist palliative care providers) and having a pre-existing IT infrastructure:

'The community SPC already used a virtual delegating system for all visits, the eShift system. ...This has been expanded during the pandemic to include all telephone consultations and reviews. This practice has allowed the team to convert to telephone consultations in a streamline manner already using the eshift structure. It has also allowed clinicians to work from home whilst still being supervised and supported clinically.' (participant 173, UK, adult, inpatient palliative care unit/home palliative care team)

A lack of access to basic IT equipment such as cameras, microphones or laptops, poor Wi-Fi or internet connection and there being too many digital platforms was a barrier to change. The need to implement remote working rapidly meant there was no time for training and staff could lack confidence and be unfamiliar with the technology but needed to learn quickly. Emergency COVID-19 funding was available but the sustainability of out of hours services without adequate funding was raised as an issue:

'7 day service as patients and families had equitable services 7 days a week. Staff felt supported and wanted this sustained. Business case for 7 day services escalated.' (participant 346, UK, adult, hospital palliative care team)

Changes were also being implemented at a time of heightened patient, family carer and healthcare professional anxiety and fear and when services needed to handle and digest ever changing information which one respondent described as an 'infodemic' (participant 107, UK, adult, inpatient palliative care unit/hospital palliative care team/home palliative care team). There was also evidence of effort duplication with services producing their own guidelines, procedures and policies.

\section{Discussion}

\section{Main findings}

Hospice and specialist palliative care services had to implement changes rapidly to respond to the anticipated and actual impact of the COVID-19 pandemic. Changes in practice involved streamlining, extending and increasing outreach of services, as well the use of communication technology and innovations for staff wellbeing. A number of barriers and enablers to change were evident such as patient, family carer and healthcare professional fear and anxiety, duplication of effort, pooling of staffing resources and collaborative teamwork.

\section{What this study adds}

Changes seen do not reflect the standard literature on the diffusion of innovations. ${ }^{6,9}$ Standard forms of innovation require planning and funding, often impossible when responding to an unforeseen event like the COVID-19 pandemic. ${ }^{10,11}$ As discussed in the introduction, the term improvisation rather than innovation has been used in crisis management, ${ }^{10-13}$ as organisations are required to be creative by using, adjusting and recombining existing resources, structures and processes to manage the impact of a crisis. ${ }^{14}$ In these circumstances, resistance to change is limited as there is an acceptance that 'normal' rules no longer apply and a collective identity develops, as seen in this study, with clinicians no longer working in professional silos and previously resisted technology being used. ${ }^{11}$ Whilst used in a different context, such limited resistance to change resonates with Klein's concept of the 'shock doctrine'30 in which extreme crises (such as COVID19) pertain the power to 'shock' systems and, in doing so, shake up socio-cultural norms to the extent that new changes - that may have been previously resisted - can be made quicker and easier than usual.

In this study, services had to rely on a 'quick fix', 'making do', being flexible and thinking in a frugal way. So called 'frugal' or 'Jugaad' innovation can challenge standard definitions of innovation. ${ }^{31}$ The aim is to provide low cost solutions to problems in environments that have resource constraints, ${ }^{32,33}$ and has been used in healthcare in economically disadvantaged communities, ${ }^{34}$ including in the context of palliative care. ${ }^{35}$

Specialist palliative care services demonstrated considerable flexibility and 'frugal' innovation, and will continue to play an important role in managing the impact of the COVID-19 pandemic. ${ }^{36,37}$ Organisations need to build flexible and resilient systems so they can be responsive to the ongoing crisis, including threats to their income as a result of an economic downturn, and any future increases in infection rates. Both national and international collaboration, and coordinated action is required to optimise resource use and avoid duplication of effort, particularly in relation to training, policies, and guideline development, while maintaining high standards of care. This need for greater collaboration was highlighted in a recent review that found a dearth of comprehensive international COVID-19 guidance on palliative care for nursing homes. ${ }^{38}$ 


\section{Strengths and weaknesses of the study}

This study is a large multinational survey of specialist palliative care services response to the COVID-19 pandemic. Free text responses provided useful insights into how and why services made changes to their routine ways of working in response to the crisis. The survey was completed by service leads so the findings may present an overly positive view of the changes made and may not reflect the views of other practitioners working within the services taking part in this study. Negative aspects of the changes made may also not have been captured due to the wording of questions in the survey. More detailed survey responses were also generally provided by those who were native English speakers. Many respondents worked within services that provided care across multiple settings. The way in which the survey was created meant that it was not always possible to distinguish between services and settings in the free text comments. The number of paediatric services included in the survey was low so the results may not be representative of children's services.

Data were collected at a single time point so how useful and sustainable the changes were has not been captured. A successful frugal innovation or improvisation may be retained but may not be useful unless there is a similar future crisis. ${ }^{13}$ Changes in practice may lead to unethical practices and negative outcomes as resource scarcity may, in some instances, simply undermine the quality of care. ${ }^{11}$ The challenge of implementing remote clinical consultations rapidly during the pandemic with limited resources, for example, has been raised $^{39}$ and how sustainable changes are beyond the pandemic without the necessary infrastructure being in place has been questioned. ${ }^{40}$ This issue is particularly pertinent to palliative care where funding for services in some countries relies heavily on charitable funding. Further qualitative case study research is planned to explore in greater depth how services responded to the pandemic and why they did or did not implement particular changes into practice, including a more in-depth exploration of the enablers and barriers to change, and whether changes were sustained and viewed as effective.

\section{Conclusion}

Specialist palliative care services have responded rapidly to the COVID-19 pandemic. Services have demonstrated considerable flexibility and relied on 'frugal innovation' when responding to the crisis. Enablers to change included collaborative teamwork, pooling of staffing resources, staff flexibility, a pre-existing IT infrastructure and strong leadership. In addition to financial support, greater collaboration is essential to build organisational resilience by minimising duplication of effort and resource use. The effectiveness of any changes made during the crisis needs continued evaluation.

\section{Acknowledgements}

This study was part of CovPall, a multinational study, supported by the Medical Research Council, National Institute for Health Research Applied Research Collaboration South London and Cicely Saunders International. We thank all collaborators and advisors. We thank all participants, partners, PPI members and our Study Steering Group. We gratefully acknowledge technical assistance from the Precision Health Informatics Data Lab group (https://phidatalab.org) at National Institute for Health Research (NIHR) Biomedical Research Centre at South London and Maudsley NHS Foundation Trust and King's College London for the use of REDCap for data capture. Sites who contributed to this work can be found in Supplemental file 5 .

\section{The CovPall study group}

CovPall Study Team: Professor Irene J Higginson (Chief Investigator), Dr Sabrina Bajwah (Co-I), Dr Matthew Maddocks (Co-I), Professor Fliss Murtagh (Co-I), Professor Nancy Preston (Co-I), Dr Katherine E Sleeman (Co-I), Professor Catherine Walshe (Co-I), Professor Lorna K Fraser (Co-I), Dr Mevhibe B Hocaoglu (Co-I), Dr Adejoke Oluyase (Co-I), Dr Andrew Bradshaw, Lesley Dunleavy and Rachel L Cripps.

CovPall Study Partners: Hospice UK, Marie Curie, Sue Ryder, Palliative Outcome Scale Team, European Association of Palliative Care (EAPC), Together for Short Lives and Scottish Partnership for Palliative Care.

\section{Author contributions}

$\mathrm{IJH}$ is the grant holder and chief investigator; KES, MM, FEM, $\mathrm{CW}, \mathrm{NP}, \mathrm{LKF}, \mathrm{SB}, \mathrm{MBH}$ and $\mathrm{AO}$ are co-applicants for funding. IJH and $\mathrm{CW}$ with critical input from all authors wrote the protocol for the CovPall study. $\mathrm{MBH}, \mathrm{AO}, \mathrm{RC}$ and LD co-ordinated data collection and liaised with centres, with input from IJH, FEM, $\mathrm{CW}, \mathrm{NP}$ and LKF. LD, CW, AO, MBH, IJH and LKF analysed the data. All authors had access to all study data, discussed the interpretation of findings and take responsibility for data integrity and analysis. LD and CW drafted the manuscript. All authors contributed to the analysis plan and provided critical revision of the manuscript for important intellectual content. IJH is the guarantor.

\section{Declaration of conflicting interests}

The author(s) declared no potential conflicts of interest with respect to the research, authorship, and/or publication of this article.

\section{Funding}

The author(s) disclosed receipt of the following financial support for the research, authorship, and/or publication of this article: This research was supported by Medical Research Council grant number MR/V012908/1. Additional support was from the National Institute for Health Research (NIHR), Applied Research Collaboration, South London, hosted at King's College Hospital NHS Foundation Trust, and Cicely Saunders International 
(Registered Charity No. 1087195). IJH is a National Institute for Health Research (NIHR) Emeritus Senior Investigator and is supported by the NIHR Applied Research Collaboration (ARC) South London (SL) at King's College Hospital National Health Service Foundation Trust. IJH leads the Palliative and End of Life Care theme of the NIHR ARC SL and co-leads the national theme in this. MM is funded by a National Institute for Health Research (NIHR) Career Development Fellowship (CDF-201710-009) and NIHR ARC SL. LKF is funded by a NIHR Career Development Fellowship (award CDF-2018-11-ST2-002). KES is funded by a NIHR Clinician Scientist Fellowship (CS-2015-15005). RC is funded by Cicely Saunders International. FEM is a NIHR Senior Investigator. MBH is supported by the NIHR ARC $S L$. The views expressed in this article are those of the authors and not necessarily those of the NIHR, or the Department of Health and Social Care.

\section{Ethics and consent}

Research ethics committee approval for this study was obtained from King's College London Research Ethics Committee (21/04/2020, Reference; LRS19/20-18541). ISRCTN16561225. Completion of survey indicated the participant had consented to the study.

\section{ORCID iDs}

Lesley Dunleavy iD https://orcid.org/0000-0002-5924-8145

Nancy Preston (iD https://orcid.org/0000-0003-2659-2342

Sabrina Bajwah (iD) https://orcid.org/0000-0001-5338-8107

Andy Bradshaw (iD https://orcid.org/0000-0003-1717-1546

Rachel Cripps (iD https://orcid.org/0000-0001-6945-5028

Lorna K Fraser (iD https://orcid.org/0000-0002-1360-4191

Matthew Maddocks iD https://orcid.org/0000-0002-0189-0952

Fliss EM Murtagh (iD https://orcid.org/0000-0003-1289-3726

Adejoke O Oluyase iD https://orcid.org/0000-0002-1506-7262

Katherine E Sleeman iD https://orcid.org/0000-0002-9777-4373

Irene J Higginson (iD https://orcid.org/0000-0002-3687-1313

Catherine Walshe iD https://orcid.org/0000-0002-4531-8608

\section{Data sharing}

Applications for use of the survey data can be made for up to 10 years, and will be considered on a case by case basis on receipt of a methodological sound proposal to achieve aims in line with the original protocol. The study protocol is available on request. All requests for data access should be addressed to the Chief Investigator via the details on the CovPall website (https:// www.kcl.ac.uk/cicelysaunders/research/evaluating/covpallstudy, and palliativecare@kcl.ac.uk) and will be reviewed by the Study Steering Group.

\section{Supplemental material}

Supplemental material for this article is available online.

\section{References}

1. Camillus JC. Strategy as a wicked problem. Harv Bus Rev 2008; 86: 98.
2. Strategic Leadership Primer. Department of Command, Leadership and Management. US Army War College, Carlisle Barracks, PA 1998: 17013-15050, https://apps.dtic. $\mathrm{mil} / \mathrm{dtic} / \mathrm{tr} /$ fulltext/u2/a430467.pdf.

3. Leong IY-O, Lee AO-K, Ng TW, et al. The challenge of providing holistic care in a viral epidemic: opportunities for palliative care. Palliat Med 2004; 18: 12-18.

4. Baregheh A, Rowley J and Sambrook S. Towards a multidisciplinary definition of innovation. Manage Decis 2009; 47: 1323-1339.

5. Schumpeter JA. The theory of economic development. Cambridge, MA: Harvard University Press, 1934.

6. Greenhalgh T, Robert G, Macfarlane F, et al. Diffusion of innovations in service organizations: systematic review and recommendations. Milbank Q 2004; 82: 581-629.

7. Zhang X, Yu P, Yan J, et al. Using diffusion of innovation theory to understand the factors impacting patient acceptance and use of consumer e-health innovations: a case study in a primary care clinic. BMC Health Serv Res 2015; 15: 71.

8. Mohammadi MM, Poursaberi $R$ and Salahshoor MR. Evaluating the adoption of evidence-based practice using Rogers's diffusion of innovation theory: a model testing study. Health Promot Perspect 2018; 8: 25-32.

9. Rogers EM. Diffusion of innovations. 5th ed. New York, NY: Free Press, 2003.

10. Hadida AL, Tarvainen W and Rose J. Organizational improvisation: a consolidating review and framework. Int J Manage Rev 2015; 17: 437-459.

11. Wiedner R, Croft C and McGivern G. Improvisation during a crisis: hidden innovation in healthcare systems. BMJ Leader 2020: leader-2020-000259. DOI: 10.1136/ leader-2020-000259.

12. Frykmer $\mathrm{T}$, Uhr $\mathrm{C}$ and Tehler $\mathrm{H}$. On collective improvisation in crisis management - a scoping study analysis. Saf Sci 2018; 110: 100-109.

13. Adrot A and Robey D. Information technology, improvisation and crisis response: review of literature and proposal for theory. AMCIS 2008 Proceedings 2008: 397, https:// aisel.aisnet.org/amcis2008/397/.

14. Lloyd-Smith M. The COVID-19 pandemic: resilient organisational response to a low-chance, high-impact event. BMJ Leader 2020: leader-2020-000245. DOI: 10.1136/ leader-2020-000245.

15. Fusi-Schmidhauser T, Preston NJ, Keller N, et al. Conservative management of COVID-19 patients-emergency palliative care in action. J Pain Symptom Manage 2020; 60: e27-e30.

16. World Health Organisation. WHO coronavirus disease (COVID-19) dashboard, https://covid19.who.int/ (2020, accessed 15 December 2020).

17. Kumaraiah D, Yip N, Ivascu N, et al. Innovative ICU physician care models: covid-19 pandemic at NewYork-Presbyterian. NEJM Catal 2020; 1: 1-6.

18. Newby JC, Mabry MC, Carlisle BA, et al. Reflections on nursing ingenuity during the COVID-19 pandemic. J Neurosci Nurs 2020; 52: E13-E16.

19. Shanafelt T, Ripp J and Trockel M. Understanding and addressing sources of anxiety among health care professionals during the COVID-19 pandemic. JAMA 2020; 323: 2133-2134.

20. Powell VD and Silveira MJ. What should palliative care's response be to the COVID-19 pandemic? J Pain Symptom Manage 2020; 60: e1-e3. 
21. Costantini M, Sleeman KE, Peruselli C, et al. Response and role of palliative care during the COVID-19 pandemic: a national telephone survey of hospices in Italy. Palliat Med 2020; 34: 889-895.

22. Woolliscroft JO. Innovation in response to the COVID-19 pandemic crisis. Acad Med 2020; 95: 1140-1142.

23. Lee $\mathrm{TH}$. Creating the new normal: the clinician response to COVID-19. NEJM Catal 2020; 1: 1-3.

24. Nembhard IM, Burns LR and Shortell SM. Responding to Covid-19: lessons from Management Research. NEJM Catal 2020; 1: 1-7.

25. Davies A and Hayes J. Palliative care in the context of a pandemic: similar but different. Clin Med 2020; 20: 274-277.

26. Oluyase $A O$, Hocaoglu $M$, Cripps $R L$, et al. The challenges of caring for people dying from COVID-19: a multinational, observational study (CovPall). J Pain Symptom Manage. Epub ahead of print 5 February 2021. DOI: 10.1016/j.jpainsymman.2021.01.138.

27. Von Elm E, Altman DG, Egger $M$, et al. The Strengthening the Reporting of Observational Studies in Epidemiology (STROBE) statement: guidelines for reporting observational studies. Ann Intern Med 2007; 147: 573-577.

28. Eysenbach $\mathrm{G}$. Improving the quality of Web surveys: the Checklist for Reporting Results of Internet E-Surveys (CHERRIES). J Med Internet Res 2004; 6: e34.

29. Ritchie J and Lewis J. Qualitative research practice. London: SAGE, 2003.

30. Klein N. The shock doctrine: the rise of disaster capitalism. London: Allen Lane, 2007.
31. Weyrauch $T$ and Herstatt $C$. What is frugal innovation? Three defining criteria. J Frugal Innov 2016; 2: 1.

32. Khan R. How frugal innovation promotes social sustainability. Sustainability (Basel, Switzerland) 2016; 8: 1034.

33. Singh R, Gupta V and Mondal A. Jugaad-from 'Making Do' and 'Quick Fix' to an innovative, sustainable and low-cost survival strategy at the bottom of the pyramid. Int J Rural Manage 2012; 8: 87-105.

34. Bajwa SJS and Takrouri MSM. Innovations, improvisations, challenges and constraints: the untold story of anesthesia in developing nations. Anesth Essays Res 2014; 8: 1-2.

35. Kumar SK. Kerala, India: a regional community-based palliative care model. J Pain Symptom Manage 2007; 33: 623627.

36. Etkind SN, Bone AE, Lovell $\mathrm{N}$, et al. The role and response of palliative care and hospice services in epidemics and pandemics: a rapid review to inform practice during the COVID19 pandemic. J Pain Symptom Manage 2020; 60: e31-e40.

37. Downar J and Seccareccia D. Palliating a pandemic: "all patients must be cared for". J Pain Symptom Manage 2010; 39: 291-295.

38. Gilissen J, Pivodic L, Unroe KT, et al. International COVID19 palliative care guidance for nursing homes leaves key themes unaddressed. J Pain Symptom Manage 2020; 60: e56-e69.

39. Greenhalgh T, Wherton J, Shaw S, et al. Video consultations for covid-19. BMJ 2020; 368: $\mathrm{m998}$

40. Wensing $M$, Sales $A$, Armstrong $R$, et al. Implementation science in times of Covid-19. Implement Sci 2020; 15: 42. 